\title{
Outline of a Dynamical Inferential Conception of the Application of Mathematics
}

\author{
Tim Räz ${ }^{\mathrm{a}, *}$, Tilman Sauer ${ }^{\mathrm{b}}$ \\ ${ }^{a}$ Fachbereich Philosophie, Universität Konstanz, 78457 Konstanz, Germany \\ ${ }^{b}$ Institute for Theoretical Physics, Albert Einstein Center for Fundamental Physics, \\ Universität Bern, Sidlerstrasse 5, 3012 Bern, Switzerland
}

\begin{abstract}
We outline a framework for analyzing episodes from the history of science in which the application of mathematics plays a constitutive role in the conceptual development of empirical sciences. Our starting point is the inferential conception of the application of mathematics, recently advanced by Bueno and Colyvan (2011). We identify and discuss some systematic problems of this approach. We propose refinements of the inferential conception based on theoretical considerations and on the basis of a historical case study. We demonstrate the usefulness of the refined, dynamical inferential conception using the well-researched example of the genesis of general relativity. Specifically, we look at the collaboration of the physicist Einstein and the mathematician Grossmann in the years 1912-1913, which resulted in the jointly published "Outline of a Generalized Theory of Relativity and a Theory of Gravitation," a precursor theory of the final theory of general relativity. In this episode, an independently developed mathematical theory, the theory of differential invariants and the absolute differential calculus, was applied in the process of physical theorizing aiming at finding a relativistic theory of gravitation. We argue that the dynamical inferential conception not only provides a natural framework to describe and analyze this episode, but it also generates new questions and insights. We comment on the mathematical tradition on which Grossmann drew, and on his own contributions to mathematical theorizing. We argue that the dynamical inferential concep-
\end{abstract}

\footnotetext{
*Corresponding author

Email addresses: tim.raez@gmail.com (Tim Räz), tilman@itp.unibe.ch (Tilman Sauer)
}

Preprint submitted to Studies in History and Philosophy of Modern Physics July 29, 2014 
tion allows us to identify both the role of heuristics and of mathematical resources as well as the systematic role of problems and mistakes in the reconstruction of episodes of conceptual innovation and theory change.

Keywords: Applicability, Mathematics, Genesis of General Relativity, Absolute Differential Calculus, Inferential Conception, Einstein, Grossmann, Ricci, Levi-Civita, Christoffel.

\section{Highlights}

- Extends and refines the inferential conception of the application of mathematics

- Examines the interaction of mathematics and physics in the genesis of general relativity

- Focus on the first application of the absolute differential calculus (tensor calculus) to general relativity

- Emphasizes the internal dynamics of mathematical theories

\section{Introduction}

The goal of this paper is to gain a better understanding of the interaction of mathematics and physics in the genesis of empirical theories, as well as to the philosophical debate of the application of mathematics in empirical science. What we intend to do is to employ, and develop, a framework for thinking systematically about the application of mathematics. We will apply this framework to an important historical case, the use of the "absolute differential calculus", what is now called tensor calculus, in the genesis of general relativity. ${ }^{1}$

The problem of understanding how mathematics is and can be applied in the empirical sciences has been discussed in the literature on the philosophy of mathematics, but until recently the debate has only been marginal in comparison to the more dominant discussion of problems associated with

\footnotetext{
${ }^{1}$ The present paper draws on Räz (2013, Part II).
} 
pure mathematics. ${ }^{2}$ One of the starting points of the debate, some fifty years ago, is a famous paper by Eugene Wigner (1960) on the "unreasonable effectiveness of mathematics in the natural sciences." Wigner formulated his astonishment in general terms, and his examples were taken from a variety of different instances of applications from different epochs. But, historically, the background for Wigner's paper, and its impact, was the effectiveness that mathematics had borne out in the first half of the twentieth century with the emergence of both the general theory of relativity and the development of quantum theory.

Although Wigner recognized the problem as a general one, in earlier times the problem of applicability did not arise as from a moment of surprise. Euclidean geometry deals with the geometry of straight lines and circles, and solves construction tasks only with the help of ruler and compass. To be sure, it gives an axiomatic treatment of the geometry of three-dimensional rulercompass space and it proves its assertions using language and symbolism. But the origin of its theorems in practical geometric experience, and the naturalness of its axioms for our physical world were all too obvious. When Hilbert stripped the geometric axioms of their direct meaning, he still insisted that, historically, geometry was a natural science. It had only evolved to a state where its concepts and results had become so firm that no one doubted their validity any more, and they could be entirely transformed into a field of mathematics.

The origins and applicability of differential calculus may be a similar case. Conceived by its creators as a general tool to describe physical motion-see Newton's term of "fluxion" for the (time-)derivative - it was meant to be a way of putting physical phenomena into a more rigid, practical, and effective mathematical representation. As argued by Ivor Grattan-Guinness (2008), a historical perspective helps to alleviate much of the "unreasonableness" of Wigner's "unreasonable effectiveness."

Barring perhaps obscure exceptions, Euclidean geometry in all its subtlety was for a long time never applied to anything else than physical geometry. This, of course, changed with Hilbert's understanding of axiomatics, and Hilbert himself used both the electrochemical series and the laws of heredity of drosophila as models for the Euclidean axioms of linear congruence; see

\footnotetext{
${ }^{2}$ For recent survey articles of the issue of applicability, see Colyvan (2009) and Steiner (2005).
} 
Sauer and Majer (2009, pp. 420-423), Hilbert (1930). More obvious was the versatility and generality of differential calculus which proved not only applicable but indispensable for almost any field of the natural sciences throughout the nineteenth century. But the Göttingen praise of the "preestablished harmony" between mathematics and physics was also most pointedly illustrated by the example of general relativity. ${ }^{3}$

Mark Steiner (1998) took the issue of applicability a step further. He argued that there is not one problem of applicability, but many problems, that have to be kept separate. ${ }^{4}$ Steiner maintains, contra Wigner, that the use of individual mathematical concepts in empirical science may not be the main puzzle. He emphasizes the philosophical problem that mathematics as a whole turns out to be so enormously successful, despite the fact that mathematics obeys anthropocentric criteria such as beauty and convenience. ${ }^{5}$

Recent discussions of applicability have shifted their focus from the discussion of problems of applicability, such as the unreasonable effectiveness, to providing an account of the various roles that mathematics may play in application to empirical problems. In the present paper, we intend to pick up on one of these accounts, the so-called "inferential conception" of the appli-

\footnotetext{
${ }^{3}$ In a talk held in Copenhagen in March 1921, Hilbert said: "The mathematician, who has noticed with surprise so often already the preestablished harmony between his own thinking and the world, is almost forced to the conception as if nature had purposely been created in such a way that in order to grasp her the deepest mathematical speculations are needed." ("Der Mathematiker aber, der schon so oft die praestabilirte Harmonie zwischen seinem Denken und der Wirklichkeit mit Staunen bemerkt, wird fast zu der Vorstellung gezwungen, als sei die Natur eigens so eingerichtet, dass es zu ihrer Erfassung der tiefsten mathematischen Spekulationen bedarf.") (Sauer and Majer, 2009, p. 387) In his lectures on the development of mathematics in the nineteenth century, Felix Klein wrote: "But the wonderful harmony, which we find between the developments of the pure mathematicians and the intellectual constructions of the theoretical physicists, is confirmed once again in an extended realm." ("Die wunderbare Harmonie aber, welche zwischen den Entwicklungen der reinen Mathematiker und den Gedankenkonstruktionen der neueren Physiker besteht, bewährt sich aufs neue auf einem erweitertem Gebiete." (Klein, 1927, p. 79)), see also his comments on Riemannian geometry and general relativity in (Klein, 1921, pp. 557-558).

${ }^{4}$ Steiner discusses semantic applicability, concerning the validity of arguments drawing on both empirical and mathematical premises; descriptive applicability, the problem of the appropriateness of mathematical concepts to solve empirical problems, and problems of analogical reasoning, reasoning that proceeds via mathematics, or the form of mathematical equations.

${ }^{5}$ Steiner discusses the discovery of the field equations of GR, one of our case studies, on pp. 94. We will turn to his argument below in section 3.3.2.
} 
cation of mathematics proposed by Bueno and Colyvan (2011). We find the basic outline of the inferential conception to be promising for our goal of a philosophical understanding of the application of mathematics. However, we will argue that a confrontation with historical case studies forces us to extend and dynamicize this account. We will outline the inferential conception and its extension to a "dynamical inferential conception" in section 2 below.

We will then explore the approach with an important example of the application of mathematics, an episode from the genesis of general relativity, in section 3. This case not only has the advantage that we can draw on detailed historical analyses for our study, ${ }^{6}$ it is also an example from the history of science where mathematics has played a prominent role in concept formation of physical sciences. More specific reasons for choosing this particular episode will be given in section 3.3

We are not claiming that the case we examine here is particularly typical, or that it warrants general lessons about how mathematics is applied. It merely serves as a first case that exhibits some important characteristics of application. It will be necessary to extend the examination of the use of mathematics to other episodes in the genesis of general relativity, and to other cases, such as the the discovery of Maxwell's equations or the history of quantum mechanics, and finally to other fields of empirical science, in order to gain general and stable insight into this issue. In short, the philosophical account will serve as a conceptual framework, which will help us to understand the case better; this enhanced understanding, in turn, will lead to a refinement of the account.

A historical approach to the issue of application and applicability has itself a historical tradition, and there are systematic reasons why the examination of historical cases is particularly fruitful. A historical approach has been part of the discussion at least since Steiner (1998) formulated one problem of applicability as a puzzle about the surprising role of mathematics in discovery; the inferential conception, as proposed by Bueno and Colyvan (2011), has been designed to capture the historical process of application. Systematically, we are not only interested in the finished product of the process of applying mathematics, we are also interested in the process itself.

\footnotetext{
${ }^{6}$ The literature on the genesis and history of general relativity is quite extensive. A good starting point are the four volumes on the genesis of general relativity edited by Jürgen Renn (2007). More specific items will be cited where pertinent.
} 


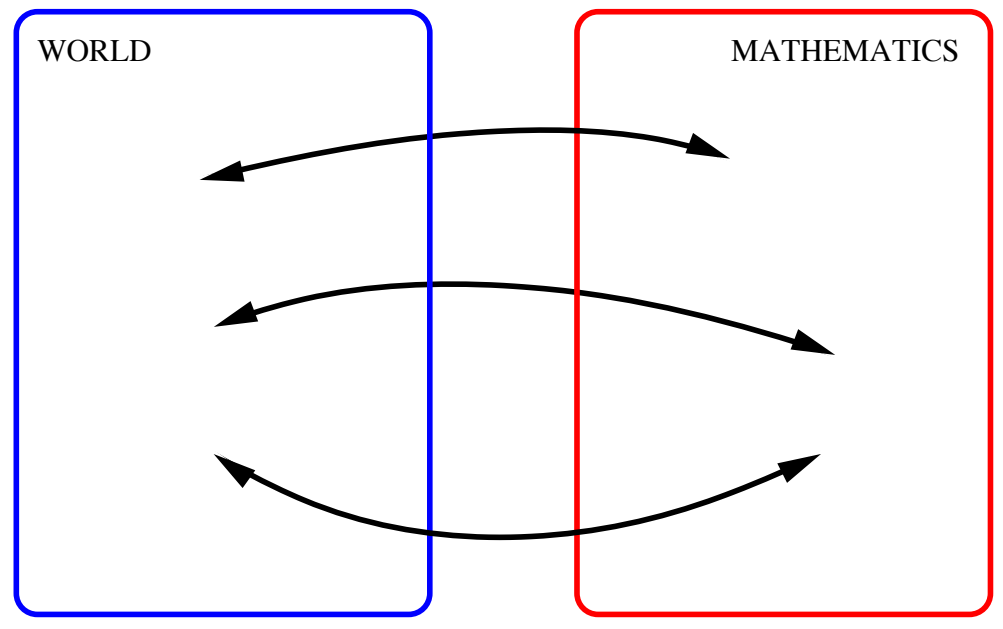

Figure 1: The mapping account: a structure-preserving mapping connects mathematical structure with relevant parts of the world.

Indeed, we are convinced that in order to fully appreciate a mathematically formulated empirical theory, it is indispensable to understand the historical process that led to this theory. We will argue that the process of applying mathematics to empirical problems plays an important role in the formation of scientific concepts, and, more generally, in theory dynamics.

\section{The Dynamical Inferential Conception}

The goal of this section is to introduce a philosophical framework for the process of the application of mathematics, which we will apply to our historical cases. We begin by sketching two existing accounts of application, the so-called "mapping account" by Christopher Pincock (2004) and the "inferential conception" proposed by Otavio Bueno and Mark Colyvan (2011), a more flexible version of the mapping account. We then discuss certain problems of the inferential conception, which motivate an extension of the approach to what we call the "dynamical inferential conception".

\subsection{The Mapping Account and the Inferential Conception}

Bueno and Colyvan (2011) use a familiar picture of applying mathematics as a foil for their own account. On this picture, mathematics helps us in application, by representing empirical structures in mathematical form; we can 
then learn about the world, by examining this mathematical representation. The application relation is established via a structure-preserving mapping, which connects the mathematical structure with relevant parts of the world. ${ }^{7}$ The mapping account is illustrated in Fig. 1.

A city street map is a useful illustration of the mapping account. A city street map represents parts of the structure of a city by mirroring the street system and buildings of the city in some detail. A map will usually leave out some information such as vertical slope, and sometimes it will not even faithfully mirror distances. However, there should be some correspondence, i.e. a mapping, between elements of the street map and elements of the city - most importantly, it should represent how various parts of the city are connected and allow inferences about actual pathways in the city. Information about the city can be inferred from information given by the map, and therein lies its usefulness.

Bueno and Colyvan (2011) identify four problems with the mapping account and argue that it cannot be a complete story of why mathematics is useful in application. The first is the so-called assumed structure problem; see section 2.2.1 below. The second problem is that the mapping account is silent on the issue of how to find the relevant structural correspondence in the first place, and to accommodate the fact that both the empirical and the mathematical domain have surplus structure. The third problem is that the mapping account appears to be unable to accommodate idealizations; see section 2.2.3 below. Finally, the fourth problem is that a static structural correspondence seems to rule out a genuinely explanatory contribution of mathematics to scientific explanations.

The motivation behind the inferential conception they propose is to solve these problems, or at least indicate how they might be solved, and thus present a more complete picture of the application of mathematics.

The inferential conception breaks down the process of applying mathematics into three steps (see also Fig. 2):

1. In the immersion step, we specify a mapping from the relevant aspects of the empirical domain to a mathematical structure.

2. In the derivation step, also called deduction step, we realize math-

\footnotetext{
${ }^{7}$ The mapping account has been spelled out in detail in Pincock (2004). Mapping accounts have a long tradition, e.g. in the debate on scientific representation and modeling. We will return to this point in the discussion of the inferential conception below.
} 


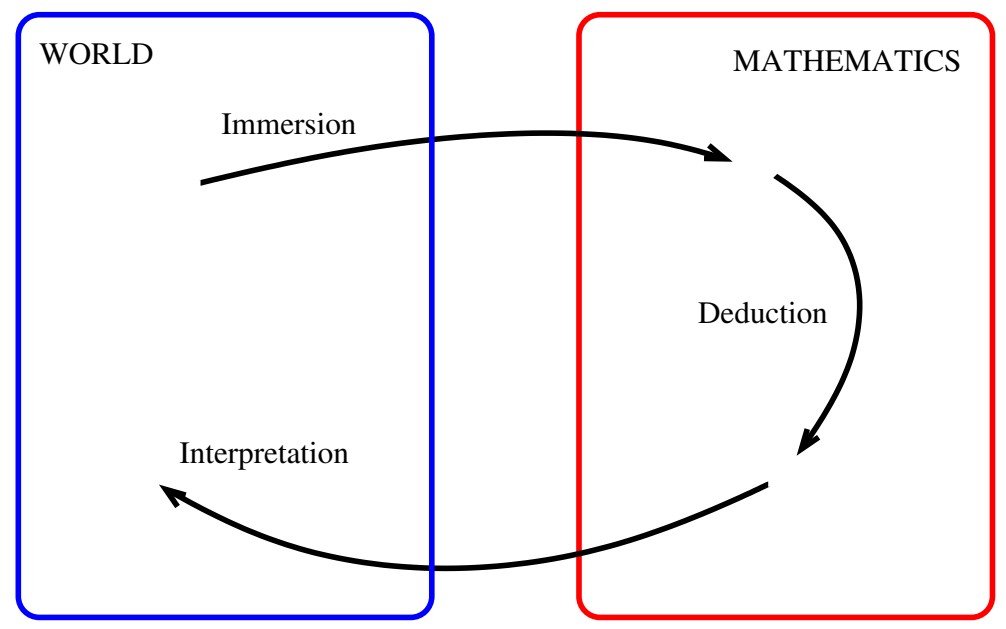

Figure 2: The inferential conception: the mapping between the empirical and mathematical domains is differentiated into an immersion step and an interpretation step, and the deduction step allows inferences licensed by the mathematical theory.

ematical inferences, licensed by the mathematical theory, about the immersed structure. This step takes place entirely within the mathematical domain.

3. In the interpretation step, the consequences found in the derivation step are mapped back to the empirical domain, that is, we interpret the results of our mathematical investigation. The mapping we use in the interpretation step is not necessarily the inverse of the immersion mapping; it need not even be of the same kind.

The inferential conception has several advantages over the mapping account.

First, since it distinguishes between the immersion and the interpretation step, different mappings may be used for the initial immersion step and the subsequent interpretation of a possibly different piece of the mathematical structure.

Second, the distinction between the immersion and interpretation steps allows us to interpret the inferential conception in a dynamical way - the suggestion is that by going back and forth between mathematics and the world, one can gradually refine the mathematical description, and also discover new empirical phenomena or revise one's assumed preconceptions about 
the world. This dynamics is a second important feature of the inferential conception. This aspect of the inferential conception is supposed to solve the second problem of the mapping account mentioned above.

A third important feature is the emphasis on the derivation step. Bueno and Colyvan (2011) describe the usefulness of mathematics in application emphasized by the inferential conception as follows: "[B]y embedding certain features of the empirical world into a mathematical structure, it is possible to obtain inferences that would otherwise be extraordinarily hard (if not impossible) to obtain." (p. 352).

Facilitating inferences is not the only role of mathematics in application. Bueno and Colyvan maintain that other functions of mathematics in application, especially unification, novel prediction, and explanation, are ultimately tied to its inferential role. They think that these aspects depend on contextual factors, and that mathematics might help to give us epistemic access to theories, by highlighting inferential patterns.

Bueno and Colyvan (2011) are not the first to emphasize the important role of the deduction, or inference step, when it comes to scientific representation and modeling. R.I.G. Hughes (1997) has proposed the "DDI" (Denotation, Demonstration, Interpretation) account of scientific modeling, which also emphasizes the inferential step on top of the world-model and the modelworld correspondences. Nancy Nersessian's (2008) and Susan Carey's (2009) accounts of conceptual change as a process of (Quinian) "bootstrapping" also consists of a trias of positing placeholder structures, modeling construction procedures such as analogy construction, limiting case analysis, thought experiments, and inductive inference, and, lastly, real world interpretation. However, there are some important differences between these accounts and the inferential conception. First, Hughes assumes that the interpretation step is the inverse of the denotation (or immersion) step - in this sense, his conception is not able to mirror the process of application. Second, his account concerns (scientific) modeling in general, not the application of mathematics. Similarly, Nersessian puts her emphasis on the inferential role of models, Carey on the role of (Quinian) uninterpreted terms and mental symbols, but neither extend their concept of bootstrapping to the specific role played by mathematics. The role of inferences in scientific modeling and representation has also been emphasized by Mauricio Suarez (2004).

Morphism-based accounts of scientific representation have been criticized; see, e.g., Suarez (2003). We will comment on what we perceive to be the most serious problems of the inferential conception in the next section. However, 
it should be noted that our topic, and our goals, are slightly different from those discussed by, say, Suarez (2003). We are not trying to formulate a general account of scientific modeling and representation in terms of morphisms; instead, our goal is to understand the role of mathematical means of reasoning in application. Also, as of now, we do not claim that our account is general; we merely hope that it will illuminate the present case, and suspend judgement as to its range of applicability until further cases are examined.

\subsection{Problems of the Inferential Conception}

In this section, we identify, and discuss, several potential problems of the inferential conception.

\subsubsection{The Assumed Structure Problem}

The mapping account, as well as the inferential conception, are based on the idea that a correspondence between mathematics and the world is established via structure-preserving mappings, usually some sort of morphism. To give an example, if we are interested in the existence of connections between parts of a city, we need a city street map that has the topological structure of the city, and we require the existence of a homeomorphism between the map and the city. This creates the following problem: If we want to account for the applicability of mathematics based on mappings, we have to assume that some sort of structure is in the world that can be preserved by the mapping. However, there is simply no guarantee that the world is conveniently structured in this way. We have to take it for granted that there is some meaningful way of discerning and using the structure of the world. This is the "assumed structure problem".

Bueno and Colyvan present a solution to this problem. If we adopt the inferential conception, we can start from a tentative assumed structure in the world, and gradually revise that structure, after our inferential investigations, by choosing a new interpretation mapping. Bueno and Colyvan (2011) write that there is no need to "formally revise" the initial assumed structure, as the interpretation mapping need not be the the inverse of the immersion mapping. Once more, the back-and-forth between empirical and mathematical domains and the emphasis on inferences are key. The assumed structure is "something like a pre-theoretic structure of the world (or at least a premodeling structure of the world)." (p. 347) As the name "assumed structure" suggests, this is not structure that we should take metaphysically seriously. Rather, it is "tentative" structure that can be revised once we have brought 
mathematics into play: "the assumed structure is the structure the modeling exercise assumes to be present in the [...] empirical setup [...] the interpretation step of the process will deliver the final structure of the empirical setup [...]" (p. 357). Thus, even if we carve the world up in a certain way prior to the immersion step, we are not committed to this being the real empirical structure of the world; the discovery of that structure is the result of going back-and-forth. The inferential conception mirrors this historical process of mathematization.

Several objections can be raised against this response of the inferential conception to the assumed structure problem. We will discuss two objections in the next paragraphs.

The Circularity Objection. A first objection ${ }^{8}$ against the inferential conception is that the assumed structure problem is an insurmountable difficulty for this approach along with all accounts that rely on mappings. We have to conceive of the immersion and interpretation mappings as mathematical objects. Therefore, both domain and codomain have to be mathematical as well. But then a mapping cannot account for the application of mathematics to the world. All we have is a mapping from an assumed mathematical structure, which is not really in the world, to mathematics. Mappings cannot possibly explain how mathematics can be applied to the world; they can only explain how mathematics can be applied to some other mathematical domain. The inferential conception is therefore circular.

This problem can be dissolved. We agree that, in some cases, including the case study we are about to consider, the assumed structure is given in mathematical form. However, this does not mean that the structure under consideration is mathematical. Rather, the mathematics represents a certain empirical structure. The immersion mapping establishes a correspondence between this empirical structure and a mathematical structure, not between two mathematical structures. As Bueno and Colyvan (2011) point out, "[i]t will be hard to even talk about the empirical setup in question without leaning heavily on the mathematical structure, prior to the immersion step." (p. 354). The immersion maps the mathematically represented assumed empirical structure to the mathematical domain.

Our second response to the circularity objection is that it runs the risk

\footnotetext{
${ }^{8}$ We thank members of the audience at the Workshop "Metaphysics of science: objects, relations and structures" of October 2012 in Lausanne for this objection.
} 
of deeming the mathematization of empirical facts inherently problematic, unless one is willing to give up on a separation between mathematics and the world, a position we discuss below in section 2.2.2. We are opposed to rejecting mathematization, as this amounts to denying a large part of modern scientific practice.

The objection nevertheless raises an important issue: we have to explain the value of basing an account of applying mathematics on mappings, because it seems that mappings can only connect mathematical domains. We will make an effort to be clear on the question as to what the starting point of the application process is, viz. how the assumed structure is constituted, and also to explain why the assumed structure is not mathematical.

The Triviality Objection. A second objection ${ }^{9}$ is to deny that there is a problem here at all, at least from the perspective of scientific realism: of course can we find objects, relations, structures in the world that are independent of mathematics and yet can be mapped onto mathematical structures. The assumed structure problem is a red herring.

This objection only has traction if we adopt a metaphysical reading of the assumed structure, i.e. if we presuppose that it is unproblematic to interpret the assumed structure as real, empirical structure that is mapped to mathematics. However, if it is the goal of, say, a new theory of gravitation to unveil the real structure of the world, but this theory is not yet available, how can we take that very structure as a starting point of our investigation? Saying that the assumed structure is just the structure of the world seems like begging the question.

When Bueno and Colyvan (2011) write about the assumed structure as the "relevant bits of the empirical world", this should not be read metaphysically. We interpret the assumed structure as the reasonable starting point of the process of applying some specific mathematics. It is not to be confused with the result of applying that mathematics.

\subsubsection{Separating Empirical and Mathematical Domain}

The inferential conception presupposes that we can draw a clear distinction between the mathematical and the empirical domain; otherwise, talk of a correspondence via mappings between these domains would not make

\footnotetext{
${ }^{9}$ We thank Matthias Egg for raising a form of this objection in the philosophy of science research seminar in the fall of 2012 in Lausanne.
} 
sense. However, it has been argued in the literature that attempts at drawing this distinction, say, in terms of the abstract-concrete distinction, are in vain, see, e.g., Ladyman and Ross (2007, pp. 159), or even that the distinction between mathematics and the world is blurred, see e.g. French (2000). This could be taken to threaten the inferentical conception, and other accounts based on mappings.

While we agree that a wholesale solution to the problem of distinguishing between pure and applied mathematics may not exist, this does not imply that the distinction can therefore be dismissed, or that no solution exists in each case. Separating the representation from what is represented is at the core of many philosophical debates, especially in the philosophy of physics. To give an example, one of the major problems that Einstein faced in the process of elaborating the general theory of relativity was the correct interpretation of coordinates. He only overcame these problems after significant struggles involving the introduction of tensorial methods and the hole argument, which turns on the question of the relation between space-time points and their representation.

This shows that, as a matter of fact, while the separation between mathematics and the world is not always clear from the beginning, it is part and parcel of the process of the application of mathematics to make it clear. Giving up on the separation of the two domains would amount to dismissing this work. This is not a viable option.

\subsubsection{Idealizations and Partial Structures}

There is one aspect of the inferential conception as proposed by Bueno and Colyvan (2011) on which we will not rely in the following, the so-called "partial structures" framework. They introduce this framework in order to solve the problem of idealization. The problem of idealization is that there appears to be no place for (deliberate) misrepresentation, if we postulate a structural correspondence between mathematics and the world. This problem, again, is common to all accounts based on mappings. Bueno and Colyvan think that all accounts that are silent on this issue should be considered incomplete.

In a nutshell, partial structures are a formal framework for structural correspondences (morphisms) that are more flexible than traditional structurepreserving mappings. Two structures that are connected by a partial morphism allow certain aspects of the correspondence to remain undetermined; consequently, these aspects are not in danger of yielding misrepresentation.

In our opinion, the main strength of the partial structures account is that 
it takes vague ideas about a partial match between empirical and mathematical structures and spells them out in a formal framework. Bueno and Colyvan (2011) introduce a case study from economics to illustrate the partial structures account. But this discussion remains at an informal level and does not exploit the strength of the partial structures account. It would be desirable to see a case study where the formal apparatus of partial structures is really put to work.

Therefore, while we agree with Bueno and Colyvan that idealizations are a challenge for any account of the applicability of mathematics, we are not convinced that the solution provided by the partial structures account can address the problem. ${ }^{10}$ But the framework of the inferential conception, on which we build, is independent of the partial structures framework. As to the problem of idealization, we will discuss particular instances as they occur, and we will pay close attention to the relation between the application of mathematics and idealization.

\subsection{The Dynamical Inferential Conception}

The inferential conception is a good starting point for a philosophical understanding of the problem of applying mathematics in the sciences, but it needs to be extended.

In this section, we introduce the extensions and modifications of the inferential conception, which we find to be necessary. These extensions are designed to accommodate a distinctively historical approach to applying mathematics, i.e. to mirror not only the result of application, but also the historical process leading to mathematically formulated theories of empirical phenomena. The extension consists mainly in a dynamization of the inferential conception. Only a dynamical inferential conception presents a conceptual framework providing us with tools to better understand historical cases. ${ }^{11}$

\footnotetext{
${ }^{10}$ See, e.g., Pincock (2005) for systematic criticism of the partial structures framework.

${ }^{11}$ Bueno and Colyvan (2011) at least implicitly construe immersion, deduction, and interpretation steps as temporal; otherwise, talk of a gradual revision process, based on going back-and-forth between mathematics and the empirical domain, would not make sense. Although we present the dynamical inferential conception as a framework for the analysis of historical cases and will illustrate and explicate our conception with a case study from the history of science, we maintain that the distinctions can also be applied to current problems of science as a framework to analyze conceptual layers of a problem of mathematization.
} 
Actual historical episodes show that certainty of success is not always presupposed when mathematical concepts, theories, and methods are applied to obtain an understanding of aspects of the real world. Where and when success was achieved, it appears from hindsight often as natural or even inevitable. But closer historical scrutiny of the episodes more often than not reveals complicated equilibration processes. These are characterized by initial misconceptions, unrecognized insights, dead ends, conceptual revisions. A systematic reason for the need to equilibrate empirical structure with mathematical structure in the process of concept formation is the fact that theories of our empirical world are necessarily grounded on and adapted to limited spheres of empirical experience. At the same time, in the process of elaborating the theory the limits of empirical validity are systematically transcended and may conflict with unexplored phenomena or with realms of the empirical world that are conceptualized by means of a different and independently obtained theoretical framework. Instances where different fields of phenomena are conceptualized in different conceptual frameworks which by no means need be compatible are discussed, e.g., by Peter Galison (1997) under the concept of "trading zones" and by Jürgen Renn's (2006) threepartite division of classical physics as the principal challenge for Einstein's early creative work.

\subsubsection{Application Cycles}

If we analyze cases of applying mathematics in the history of science, the smallest unit that is iterated in temporal succession consists of immersion, deduction and interpretation steps, as laid out in the inferential conception. We call such a unit a cycle. A cycle is one round of going back-and-forth, from the world to the mathematics and back to the world, one round of assimilating a mathematical theory and a particular empirical structure. The starting point of a cycle is the initial assumed structure, the end point is the confirmed or revised assumed structure.

We conceive of the dynamical inferential conception as an account of application of mathematics, not an account of successful application of mathematics. The framework encompasses both successful and unsuccessful applications. Thus, there are two possible outcomes for cycles. We have a closed cycle if the application is successful. On the other hand, if the application fails, we are dealing with an open cycle (see also Fig. 3).

The question whether a cycle is closed or open, i.e., the notion of success, is relative to the various goals and expectations of the scientists. Researchers 

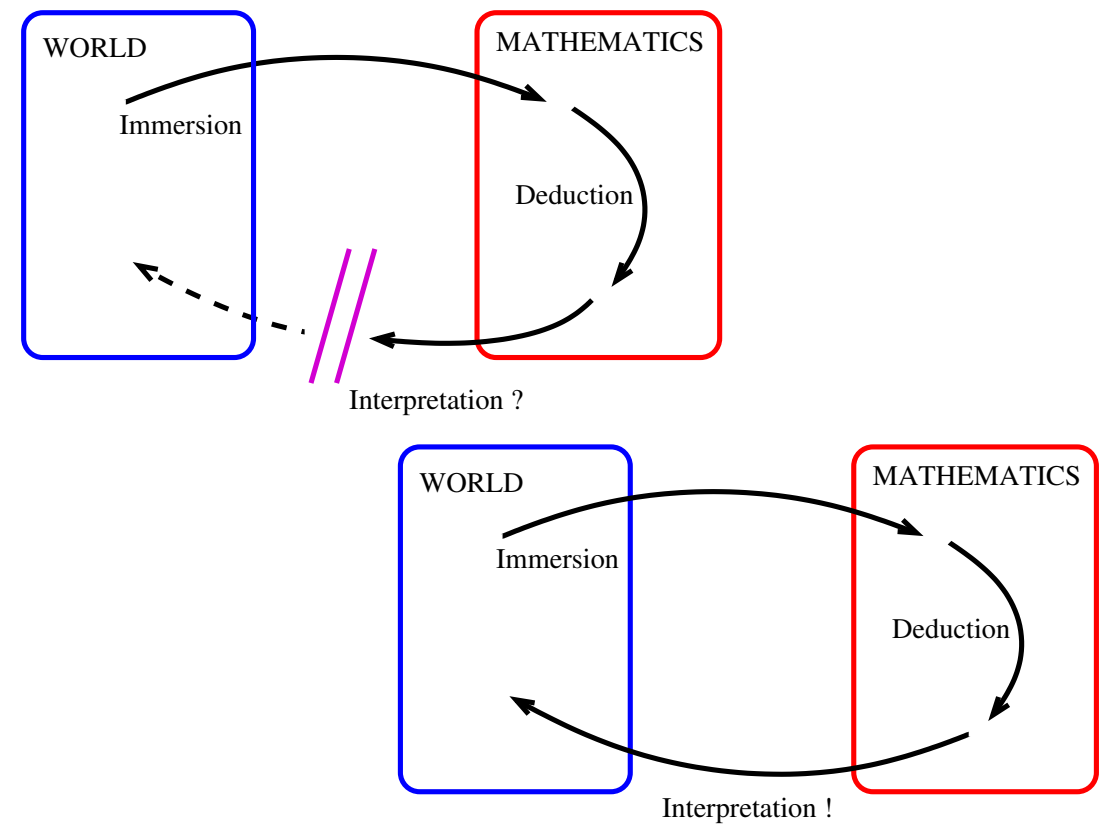

Figure 3: In the dynamical inferential conception we distinguish between open and closed cycles of applying mathematics. 
might want to understand a new empirical finding in an established theoretical framework, they might want to explore novel implications of a theory, they might want to establish links between parts of the conceptual structures, or explore known propositions and statements of the theory with respect to their logical dependencies, consistency, or completeness.

The application cycle will often be triggered by a problem that the scientist identifies in the assumed structure. This problem will also prompt a heuristics, which guides the course of the cycle. The result of an application cycle will be compared with the expectations of the solution. It is here that contradictions between expected results and derived results may manifest themselves. It is also possible that while the result of a cycle matches with expectations, other parts of the cycle, say, the derivation step, do not conform with the heuristics.

Once the goal of an application cycle is set, there is a sense in which the success, or failure, of an application cycle is independent of the scientist's persuasion. For example, there can simply be a match or mismatch between a mathematical structure and the empirical structure it is applied to. We will call this an objectively closed, or open, cycle. On the other hand, a scientist can be persuaded, rightly or wrongly, that a cycle is open or closed. For example, a scientist can commit a mistake in a calculation which goes unnoticed, and consequently be persuaded that a cycle is open, even if the cycle could be closed, were one to correct the mistake. We will call this kind of cycle subjectively closed or open. This distinction is particularly important in order to understand the historical genesis of a mathematized theory. We will point out what kind of cycle we are dealing with if necessary.

Depending on the nature of the problem, and on the outcome of the application cycle, different dynamics are triggered. If the outcome is an open cycle, i.e., the scientist finds that there is a mismatch between the mathematically derived result and the empirical domain, a process of reflection is set in motion: The entire pathway of the open cycle is being reconsidered. The analysis of the cycle is no longer exploratory, but the cycle is reflected on with a view of identifying the points of failure for the open cycle.

The failure of the cycle may have occurred at any one, or several, of the steps along the cycle, and we can use the components of the cycle as proposed in the inferential conception as a diagnostic tool to analyze open cycles, and potentially to overcome the difficulty. The process of engaging in an application cycle in exploration mode and thereafter in reflection mode captures real moments of the research process, but these categories also pro- 
vide conceptual tools to analyze the historical process, even if the actors did not perceive their actions in those terms.

There are thus four categories of problems that can underly an objectively open cycle, i.e. an application cycle which is unsuccessful relative to a set goal. These problems correspond to the four components of a cycle.

1. The failure can be due to an inadequate initial assumed structure. Maybe the empirical phenomena taken as the starting point of the modeling process have to be investigated more closely, and more data have to be collected. Maybe it is even necessary to find a different conceptualization of the empirical phenomena.

2. The failure can be due to the immersion step, or the mathematical theory we use. The framework may lack the expressive power for a task, it can be insufficiently understood, or it can exhibit internal difficulties, or even inconsistencies. In this case, the problem can be fixed, either by revising, or further exploring, the mathematical framework, or it could be given up entirely.

3. There could be a failure in the derivation step. Certain inferences can be hard, or even impossible, to reach, so that it is hard to reconnect the findings of the deduction step with the world in the interpretation. In this case, the scientist could make an additional assumption to facilitate the deduction, search for a different framework, or explore alternative routes of deduction.

4. There could be a failure in the interpretation step. For example, it could be unclear whether a solution to some equations has to be interpreted realistically, or if it is just a mathematical artifact. It may also be the case that elements of the mathematical framework are misinterpreted in their proper reference to elements of the empirical structure.

However, a cycle can also be only subjectively open, for example if the scientist committed a mistake somewhere along the way. One such case occurred during Einstein's search for a differential operator for the field equations of general relativity. Again, the dynamical inferential conception provides a framework for thinking systematically about application mistakes: we can locate the mistakes in the components of application cycles. Here are the different kinds of mistakes that one can commit in a subjectively open application cycle:

1. Assumed Structure Mistake: This mistake occurs when there are wrong expectations about the starting point of an application cycle, or 
when there are wrong expectations about the empirical target structure one expects to recover when completing a cycle.

2. Immersion Step Mistake: This is the mistake of taking some empirical phenomenon and choosing an unsuitable mathematical representation for it. It can happen that we have a clear mathematical counterpart for one empirical object, but that it is unclear as to what the appropriate representation of other aspects of reality will be. For example, knowing that the line element represents distances between space-time points does not solve the problem of how to represent points of space-time.

3. Deduction Step Mistake: These are mistakes that occur in the deduction step, such as errors in calculations or the failure to fully exploit the deductive possibilities of a mathematical theory.

4. Interpretation Step Mistake: This is the mistake of interpreting part of the mathematical formalism a) realistically, if the mathematical object has properties that are purely representational, or b) not realistically, if it allows for a realistic interpretation. An important example for our case study are coordinate systems: For a long time, Einstein interpreted them realistically, before he realized that they are, in fact, mere tools of representation.

If an application cycle is closed, the process of application is not over, but a different kind of dynamics is set in motion. Usually, a closed cycle only means that the scientist has successfully derived some consequences within mathematics that have a meaningful empirical interpretation, not that there is a full match between the mathematical and empirical structure. The further goal will normally be to consolidate the closed cycle. Again, improvements are possible in all components: the scientist can send the revised assumed structure back to mathematics in the second cycle, or even widen the scope of phenomena in the assumed structure, and check whether the cycle can still be closed; one can work out further deductions and interpretations from the original immersed structure; the validity of the deductions can still be checked; and, the interpretation can suggest further empirical investigations, if it yields novel predictions that have not been anticipated, and so on. 


\subsubsection{The Internal Structure and Dynamics of the Two Domains}

So far, we have characterized the inferential conception with a focus on the mappings between the empirical and the mathematical domain, on derivations on the mathematical side for the sake of application, and on how these steps shape the two domains. By adopting this perspective, we run the risk of construing the two domains as passive entities that are exclusively shaped by their interaction.

The examination of the historical cases confirms that this picture is too narrow. The two domains evolve over time as a result of processes of equilibration between physics and mathematics, but also autonomously. We thus advocate a dynamic picture of domains. This dynamization is compatible with the original inferential conception; it extends the view. We will see in our case study on the general theory of relativity, in section 3.3.3, that notably the mathematical domain is a dynamical entity.

The fact that the mathematical domain may have a dynamics of its own draws attention to its internal structure. By this we mean that mathematics comes in different layers of theories, where one mathematical theory is more abstract than the other, and we can apply the former to the latter. This suggests that we get application cycles of a different kind, which are located at the level of pure mathematics. We will describe one such cycle in the case study on general relativity below. We should emphasize that while there are parallels between the internal application of mathematics and the application of mathematics to the world, there are good reasons to keep these cases separate. The most important difference between the two cases is that there is no assumed structure, and no assumed structure problem, in mathematics; there are only morphisms between mathematical structures.

Examining the internal dynamics of mathematics forces us to be specific about the mathematical theory that is applied, both within mathematics and to the world. Formulating the problem of applicability of mathematics in generic categories of mathematics and the empirical world presents the problem in a way that is too coarse-grained. Each and every case of applying mathematics is a case of applying a specific piece of mathematics. Also, it should be noted that even if the kind of morphism used in the immersion or interpretation step is specified, this need not determine the mathematical theory that is applied, especially if it is possible to interpret one mathematical theory in terms of another - think of a calculus with and without "intended interpretation." 


\section{The Case of General Relativity}

We now put the dynamical inferential conception to work in our historical case study, an episode from the genesis of the general theory of relativity. We will first sketch the state of play at the beginning of the search for GR, in the period before the absolute differential calculus (ADC) of Tullio LeviCivita and Gregorio Ricci-Curbastro (1901), and provide a short overview of the evolution of the theory up to that point. We will then reconstruct the application of this new kind of mathematics drawing on the framework of the dynamical inferential conception.

\subsection{Initiating the Search for a Relativistic Theory of Gravitation}

The search for a new-or revised - theory of gravitation was triggered by the conflict between special relativity (SR), which postulates a finite, constant speed of light $c$, and Newtonian Gravitational Theory (NGT), which implies an instantaneous propagation of gravitational effects. These two conceptualizations of partly distinct physical domains were inconsistent and had to be reconciled.

While the inconsistency is rooted in physical principles, it carries over to a formal inconsistency in the respective mathematical formulations of the two theories: NGT in its received form does not conform to the formal requirement imposed by SR, Lorentz covariance. Therefore, the mathematical formulation of the theories had to be adapted. This set the process of the application of mathematics in motion. However, it was not clear whether radical changes in the mathematics, or the application of a new kind of mathematics were necessary, or whether a more conservative revision of NGT was sufficient.

Historically, various theoreticians were engaged with this problem in the period between the establishment of SR and the advent of GR, i.e. in the decade between 1905 and 1915. There were proposals by Hermann Minkowski and Henri Poincaré to find force laws that would be Lorentz covariant and exhibit a finite propagation speed of electrodynamic interaction, as well as attempts to formulate field theoretical modifications of gravitation, as proposed, e.g., by Max Abraham; see Renn and Schemmel (2007a,b). ${ }^{12}$

\footnotetext{
${ }^{12}$ Other relevant developments were oblique to these dates and events, e.g. attempts at modifying the theory of gravitation pre-1905, motivated by finding a field theory of gravitation in analogy to electrodynamic field theory, or by finding modified force laws,
} 
The historical starting point of Einstein's investigations were the two theories that triggered the search for a relativistic theory of gravitation: SR on the one hand, and NGT on the other. Within the framework of the dynamical inferential conception, these two theories capture the initial assumed structure at the beginning of the application cycles leading to GR. The initial assumed structure thus already came in mathematical form. This was to be expected, because once physical theories have reached a certain degree of sophistication, we do not start from scratch, but we pick up the construction of the theory mid-stream. ${ }^{13}$

At this point, we want to be careful to keep apart the assumed structure, i.e. the empirical phenomena described by a theory, and the mathematical formulation of the same theory. At the same time, the theories do not capture all relevant empirical phenomena - this motivates the distinction between empirical domain and assumed structure. Additionally, the different theories constituting the assumed structure exhibit inconsistencies, and play different roles in the heuristics of the application process. We will now spell out these points for the case at hand.

First, what empirical phenomena are captured by the two theories? Special relativity arose from a reflection on properties of electrodynamic field theory. It was then generalized to a restriction on any physical theory, such as thermodynamics or classical mechanics. The restriction was formulated in terms of restrictions on the mathematical formulation of physical theories: Their laws had to be formulated in a Lorentz covariant way. Newtonian gravitation theory, on the other hand, had been a foundational theory for centuries with an elaborate mathematical formulation. But with the advent of electrodynamic field theory, it became a theory of a restricted domain of physical phenomena. It deals with the motion of massive bodies moving in space under the influence of the gravitational force, such as the motion of the planets around the sun, and falling bodies on earth.

There were two lines of conflict between the two theories. First, NGT was well confirmed for small velocities. This was not true for velocities approaching the speed of light, where the inconsistency with SR may become apparent. Second, some isolated gravitational phenomena, in particular the

motivated by electrodynamics force laws (Weber-like laws), or motivated, in either case, by empirical anomalies (Mercury perihelion advance); see Renn and Schemmel (2007a,b).

${ }^{13}$ The fact that the assumed structure may come in mathematical form has already been pointed out by Bueno and Colyvan (2011); see the discussion in section 2.2.1 above. 
anomalous perihelion advance of Mercury, could only be explained within the theoretical framework of NGT by imposing ad hoc assumptions. It was known for quite some time that NGT could not account for this anomaly, which is also a gravitational phenomenon; see Newcomb (1895); Rosevaere (1982).

These two lines of conflict did not yield equal reactions. The first shortcoming was at the center of Einstein's attention. The conflict between SR and NGT triggered the search for GR, and it was one of the main goals of the new theory to remove the internal inconsistency between the two theories. The second line of conflict did not trigger the construction process of general relativity. The empirically observed motion of Mercury - the anomalous advance of its orbit - was part of the empirical domain, but it was not part of the assumed structure. It could not be reproduced by applying the mathematics of NGT. The assumed structure thus contained a part that would be interpreted as Mercury's orbit (a deduced function) but that function was known to be inconsistent with empirical data (numerical values empirically associated with the arguments of the deduced function). The mismatch between the theoretically obtained planetary orbit, which was part of the assumed structure, and the empirically observed orbit, which was part of the relevant empirical domain, played a different role in the application cycle. The function of the empirical observation was that of a touchstone, it entered the construction process as part of the heuristics. One criterion for a successful application cycle would be to see whether the mathematical structure, into which one would map the assumed structure in the immersion step, allowed one to account for the correct numerical value of the perihelion advance, after mapping back to the empirical domain in the interpretation step; see Earman and Janssen (1993). The secular advance of the perihelion of a planet's orbit both gives deductions in the mathematical domain a goal and direction and it provides an empirical value that may or may not be recovered in the application cycle.

NGT was the part of the initial assumed structure where the inconsistency was located, both in its empirical falsification and in its theoretical competition with special-relativistic electrodynamic field theory. NGT became the part of the assumed structure that was perceived to be in need of revision. There are systematic reasons for this starting point. Those reasons have to do with the heuristic "correspondence principle," one of the guiding requirements identified by Renn and Sauer (1999) as shaping the search for GR. According to the correspondence principle, the new theory should repro- 
duce NGT in a special-relativistic, weak-field limit, such that the empirical knowledge embodied in NGT would be recovered. The heuristic requirement of a Newtonian limit therefore fulfilled a function of utmost importance: it guaranteed that the new theory would be empirically adequate, at least to the same extent as NGT, and the empirical adequacy of the new theory would not have to be demonstrated from scratch. Building the old theory into the new one was not only a theoretical requirement: The specification of a certain limit for the recovery of NGT opened up the possibility of revising the assumed structure in regions where the premises of that limit would no longer hold.

This concludes our characterization of the initial assumed structure of general relativity. Now we will sketch how the initial assumed structure was refined before the absolute differential calculus came into play.

\subsection{The Genesis of General Relativity: A Drama in Three Acts}

Following John Stachel (2007), we can conceive of the genesis of GR as a drama in three acts. In the first act, Einstein (1907) formulated the "equivalence hypothesis," the assumption that there is a complete physical equivalence between a frame of reference moving in linear, rectilinear relative acceleration to some inertial frame, and an acceleration-free frame endowed with a static, homogeneous gravitational field. The equivalence hypothesis was a heuristic tool that allowed Einstein to analyze properties of gravitational fields using coordinate transformation between moving frames of reference. He derived three physical consequences of his hypothesis: gravitational red shift, gravitational light bending, and the gravitational mass of energy. In the second act, Einstein realized that a proper mathematical representation of a relativistic theory of gravitation would have to be based on a second-rank, symmetric tensor field, the metric, which expressed properties of space-time and of the gravitational potential. In order to fulfill this function, the metric had to be non-Euclidean. The fact that the metric would play a prominent role prompted the question as to how it would be determined by a relativistic field equation; this is the starting point of act three. Fortunately, we have insight into act three, the search for the relativistic field equation, by means of a most significant historical document, Einstein's Zurich notebook, which has been subject of intense historical investigation; see Renn (2007). For a while, Einstein settled on a system of gravitational field equations that is not generally covariant, and worked in a state of suspense, until he finally 
achieved the breakthrough to full general covariance in late 1915, in hectic competition with the world's leading mathematician, David Hilbert; see Sauer (1999).

The episode on which we will focus here belongs to the third act, the search for a relativistic field equation. However, before zooming in on an analysis of this episode in terms of the dynamical inferential conception, we want to add some comments on the first two acts.

In the first act, sophisticated mathematical concepts and methods were introduced by other protagonists, notably by Minkowski (1908), who reformulated the original theory of SR in a formal, four-dimensional representation, drawing on mathematical traditions of matrix theory introduced by Arthur Cayley. Initially, Einstein was sceptic towards these mathematical reformulations, until he was confronted with their advantages in an competitive encounter with Max Abraham, one of the proponents of sophisticated mathematical methods in theoretical physics. ${ }^{14}$

For our purposes, two results of the encounter are significant. First, it made Einstein realize the expressive and inferential power of mathematical structure in theory construction. Second, more concretely, it showed that, in the context of a relativistic theory of gravitation based on the equivalence hypothesis, the Minkowski light-cone invariant had to be taken in differential form if the velocity of light $c$ was no longer a constant. As a further consequence, it became clear that the differential line element with variable $c$ actually transcended Lorentz covariance. However, this insight also paved the way for the use of a general differential line element. This would prove to be significant in the second act, where the metric tensor suddenly appeared on stage as major character for the remainder of the drama.

Another important development of the first act concerns the representation of the source of the gravitational field. In NGT, the source is the mass density, a scalar field. Again, physical and mathematical considerations in the elaboration of SR transformed the source of the gravitation field into a second-rank symmetric tensor, the stress-energy-momentum tensor, comprising mass-energy density, momentum flux, as well as stresses as physical quantities which all contribute to the generation of a gravitational field.

With the appearance of the metric tensor, on the one hand, and the

\footnotetext{
${ }^{14}$ The encounter with Abraham can itself be analyzed in terms of the dynamical inferential conception; see Räz and Sauer, manuscript in preparation.
} 
energy-momentum tensor, on the other, the classical Poisson equation, which defined their relationship, had to be transformed as well. This is what triggered the dynamics of the episode to which we will now turn.

\subsection{The Absolute Differential Calculus: A New Kind of Mathematics}

In this section, we apply the dynamical inferential conception to one of the most interesting episodes in the genesis of GR, the collaboration of Einstein with his "mathematician friend", Marcel Grossmann, on the so-called Entwurf ("outline") theory of gravitation in 1912-13, the immediate precursor theory of final general relativity. ${ }^{15}$ The collaboration began at some time in late summer 1912 when Einstein tried to generalize the equivalence principle to reference frames of arbitrary acceleration, but faced mathematical difficulties. Legend has it that in a state of desperation, he turned to Marcel Grossmann, his good friend from student days and now professor of geometry at the ETH Zurich. Grossmann identified the absolute differential calculus (ADC), an early version of tensor calculus, as a mathematical theory that could, perhaps, solve Einstein's problems. Together, Einstein and Grossmann reformulated gravitational theory in this new framework, and published the first tensorial formulation of GR, which champions all essential elements of the final theory of general relativity, except for the correct gravitational field equations.

The episode is a particularly interesting case of the application of mathematics for the following reasons. First, the mathematics applied in the Entwurf, the ADC, was developed independently of the particular physical problem at hand, gravitational theory. This sets it apart from the previous application cycles; we will elaborate on the peculiarities of this aspect of the cycle in section 3.3.3 below. Because of this feature, it has been discussed as an example of the "unreasonable effectiveness" of mathematics; see Steiner (1998). Secondly, at this stage of history, the division of labour between mathematics and physics is discernible at several levels. The protagonists of the episode, Einstein and Grossmann, both had clearly defined competences and tasks: Einstein initiated the collaboration and brought the physical mo-

\footnotetext{
${ }^{15}$ The Einstein-Grossmann theory is often referred to in the literature as the Entwurf theory with reference to Einstein's and Grossmann's first joint publication, entitled "Outline (Entwurf) of a generalized relativity theory and a theory of gravitation" Einstein and Grossmann (1913). For a detailed account of their collaboration and Grossmann's role in it; see Sauer (2014).
} 
tivation and knowledge to the table. Grossmann's competence was in finding a theory that solved a clearly-formulated mathematical problem. This division of labour carries over to the resulting joint publication. The Entwurf paper has two parts: Einstein was responsible for the first, physical part, while Grossmann was responsible for the second, mathematical part. Third, the case is interesting because Einstein and Grossmann were not yet able to carry out the application process to their satisfaction, as the main application cycle remained open. The characterization of the theory as an Entwurf proved to be justified, as the Entwurf field equations turned out to be wrong in the end.

\subsubsection{Assumed Structure}

Since the inception of the drama of GR in 1907 with the formulation of the equivalence hypothesis, the assumed structure had been substantially revised when Einstein and Grossmann began their collaboration in 1912. Einstein's goal, at the beginning of act three, was to find relativistic field equations of gravitation, i.e. an equation of the form

$$
O P(P O T)=S O U R C E .
$$

This is a so-called "frame," a template that can be instantiated in different ways depending on the context; see (Renn and Sauer, 2007, p. 127). Its instances are differential equations which determine a potential (POT) from a distribution of sources (SOURCE) by way of a differential equation which arises from a differential operator (OP) acting on the potential. Examples are the electrostatic Poisson equation, determining the electrostatic potential $\varphi_{e}$ as a function of the charge density $\rho_{e}$, or the inhomogeneous wave equation for the electromagnetic four-potential $A^{\mu}$ where the source is given by the fourcurrent $j^{\mu}$. At the beginning of the search for GR, the frame was instantiated by the gravitational Poisson equation, at its end it would be transformed into the Einstein equations. In 1912, two of the three components of the frame had been generalized. The SOURCE slot was filled with the energy-momentum (EM) tensor $T_{\mu \nu}$, and the POT slot was filled with the space-time metric $g_{\mu \nu}$. The remaining task was to find, and examine, suitable candidates for OP, the differential operator acting on the metric.

\subsubsection{Immersion Mapping}

The immersion mapping connects the initial assumed structure with the mathematical domain. In this case, the immersion hinged on a reinterpreta- 
tion of the differential line element. The expression

$$
d s^{2}=g_{\mu \nu} d x^{\mu} d x^{\nu}
$$

where $g_{\mu \nu}$ denotes the metric tensor, had been generated in the physical domain as a result of previous application cycles. By embedding it into the mathematical domain, it was now being stripped of its physical significance, and reinterpreted as the symbolic expression of a differential line element. This change of perspective established a connection to the theory of differential invariants. The goal of the immersion step was to find differential operators that could enter into the field equation. The mathematical object that had to be carried along in the immersion, to be generalized in the mathematical domain, was the Laplace operator. Thus, the main task was to find differential operators acting on the $g_{\mu \nu}$. The physically motivated heuristics translates into the search for a mathematical theory that provides covariants of a homogeneous quadratic differential form.

Einstein's formulation of the task in the Entwurf paper makes it clear that the requirements on a suitable differential operator are tentative in character and potentially subject to revision:

In accordance to [the Poisson equation], one is inclined to require that [the new relativistic field equation] be of order two. However, it has to be emphasized that it proved to be impossible to find a differential equation that satisfies this requirement, is a generalization of [the Laplace operator], and is tensorial for arbitrary transformations. A priori we cannot exclude that the final, exact equations of gravitation are of order bigger than two. [...] The attempt of a discussion of such possibilities, however, would be premature in view of our present knowledge of the physical properties of the gravitational field (Einstein and Grossmann, 1913, p. 11).

We can extract a list of requirements, Einstein's "check list" for differential operators, from the above quote:

1. The operator should be of order two.

2. It should be invariant under transformations larger than the Lorentz group.

3. It should be a generalization of the Laplace operator. 
The first is a heuristic requirement derived from the Poisson equation. It had no conclusive justification, but was based on an analogy with the classical case. The second is based on the principle of "generalized relativity". ${ }^{16}$ The third, finally, is grounded in the correspondence principle.

Let us comment briefly on the first requirement. Steiner (1998, pp. 94) discusses the use of analogies in the discovery of the equations of GR. His main point of contention is that "the equation should be a second degree differential equation, and linear in the second derivatives." Citing Graves (1971, p. 178), he claims that there is no physical justification for this choice, but these conditions were simply adopted from the Poisson equation. He claims that "the analogy with the Poisson equation was a Pythagorean analogy". A Pythagorean analogy is an analogy which proceeds by using some mathematical property of an equation, for which there is, at the time of the discovery, no physical justification. This, however, seems to be overreaching. First, the use of the Poisson equation is justified by the correspondence principle. Furthermore, some properties of the Poisson equation do play a role here. The associated Green's function maps back to the Newtonian gravitational potential, and for physical reasons, one would expect second-order differential equations of motion to hold, which require initial conditions of position and velocity, and nothing more. Therefore, in the absence of theoretical reasons to go beyond second-order terms, there is no physical reason to look for higher-order differential equations, either on the level of the equations of motion or the level of the field equation.

Most of the specifications for the differential operators were extracted from the existing mathematical formulation of NGT, the Poisson equation. The requirement for a wider covariance group, on the other hand, was new. Unfortunately, the available historical sources concerning this issue are much too vague on this point: We do not know how exactly Einstein formulated the request for candidate differential operators when he approached Grossmann for help, so it is not clear whether he thought that general covariance was required, or if he had only a vague idea that the covariance group should be

\footnotetext{
${ }^{16}$ This principle is closely related to the equivalence principle. It suggests generalizing to non-inertial frames the idea from SR that there are no privileged inertial frames. In this sense, the principle has to do with the question as to which properties of space-time should be accepted a priori. In Einsteins mind, the requirement to generalize SR was closely related to finding an appropriate mathematical description of gravitation that was independent of the chosen coordinate system.
} 
larger, and Grossmann filled in the details later on the bases of the ADC. ${ }^{17}$ There are later recollections of the precise formulation of Einstein's question, but such later accounts, as always, have to be taken with a lot of caution.

\subsubsection{The Mathematical Domain}

The target of the immersion step is the mathematical domain, one or more mathematical theories with more or less worked out rules of inference embodied by rules of transformation and manipulation for symbol systems. The mathematical domain helps representing the structure immersed into it, but it can have surplus structure. The mathematical domain should also not be conflated with the deduction step. The mathematical domain is a general framework which provides a space of possible structures and deductions, while the deduction step is geared towards a specific goal of application, putting mathematical theories to use.

According to the inferential conception, the next step in the application process is the derivation step: once a part of the empirical domain is represented as a mathematical structure, we use a mathematical theory to gain knowledge about this structure. However, we think that at this junction, the inferential conception in its simple form is too coarse-grained and requires further refinement. It leaves out important aspects of the application process. It may not be possible to simply use an existing mathematical theory in the derivation step, as sometimes, a considerable effort is involved in making a mathematical theory applicable at several levels.

This point emerges from a closer look at the present episode. The main difference between the previous application cycles and the present one is that here, an existing, more or less worked out mathematical theory, the ADC, is put to work in the context of gravitation for the first time. The novel application of a mathematical theory is one of the more puzzling aspects of applicability, so we want to be particularly careful in our examination of how it works. ${ }^{18}$

A mathematical theory can be more or less suitable for a particular application, it can contribute to its own application more or less successfully, and it can be modified so as to enhance its applicability. It is fruitful to dis-

\footnotetext{
${ }^{17}$ See Sauer (2014) for a detailed discussion of this question.

${ }^{18}$ Steiner (2005, Par. II) calls this "noncanonical empirical applications", empirical application in a context for which the mathematics was not devised; Steiner classifies the application of non-Euclidean geometry in GR as noncanonical.
} 
tinguish two aspects of the contribution of a mathematical theory towards application: It can be implemented before, and after, the mathematical theory is actually applied to a particular empirical domain. We will call the first case "prior", and the second case "posterior" contributions of mathematics to application.

The "prior" contributions of mathematical theories to application are those aspects that are regulated by considerations within pure mathematics, and general considerations about the potential application of the mathematical theory by mathematicians, prior to the application of the mathematical theory to any particular empirical domain. In order to understand the prior contributions in the present case, we have to examine the evolution of the mathematical theory before its application to gravitation.

The "posterior" contributions, on the other hand, have to do with the adaption of the mathematical theory to one particular application. They are not part of the derivation step, properly speaking, but prepare the ground for this step. In the present case, Grossmann's contributions pertain to this aspect. We will ask: Did Grossman adapt, change, or extend the existing mathematics in order to make it applicable? Did he make original contributions to the applied mathematics?

The Mathematical Theories in the Entwurf. Before we turn to a discussion about the prior and posterior contributions of mathematics, we have to determine which mathematical theories are applied in the Entwurf, and what the relative importance of these theories and traditions is. ${ }^{19}$

In the mathematical part of the Entwurf, Grossmann cites the following works: Christoffel (1869), Bianchi and Lukat (1899), Riemann (1876), Ricci and Levi-Civita (1901), Kottler (1912). In addition, there are references to Minkowski (1908), Sommerfeld (1910a,b), and Laue (1913). The latter are formal reformulations of special relativity using four-dimension notation and four-dimensional vector algebra and calculus. Although these researchers have a background in mathematics, their works are not themselves mathe-

\footnotetext{
${ }^{19}$ The question as to what the most important mathematical tradition for the genesis of GR was, has been debated in the historical literature; see e.g. Reich (1994); Stachel (2007). Räz (2013, Sec. 5.2.3) is a very short introduction of the "new" mathematics applied in the Entwurf theory, and Räz (2013, Ch. 6) discusses the influences of the mathematical sources on the Grossmann's part of the Entwurf. In the following, we will draw on these works, as well as the original sources.
} 
matical. We therefore concentrate on the references that cite genuine mathematical contributions. It is notable that Abraham's work is not explicitly mentioned.

Not all of these mathematical sources are equally important, as was argued in detail in Räz (2013, Ch. 6). Based on Grossmann's citations, and on a comparison of the notation used by Grossmann and the mathematical sources, the works by Christoffel, Bianchi, and Ricci and Levi-Civita stand out as particularly important. Kottler's work is probably mentioned only to avoid a priority dispute. Finally, an analysis of Grossmann's reference to Riemann suggests that Grossmann did not consult Riemann's work directly, and that the latter's influence was much less important than talk of the role of Riemannian geometry for GR suggests. ${ }^{20}$

The influence of Ricci and Levi-Civita (1901) on Grossmann and Einstein is well known: Ricci and Levi-Civita provided Grossmann with a worked out calculus, on which he drew freely. Their paper has survey character, and Grossmann may have used it as an entry point into the mathematical problems at hand. On the other hand, the importance of Christoffel (1869) for Grossmann cannot be overemphasized. To give but one example, Grossmann did not use the notation provided by Ricci and Levi-Civita for the most part, but he went back to Christoffel's notation. A parallel reading of Grossmann, Christoffel, and Bianchi is also instructive. Bianchi and Lukat (1899) is a textbook on differential geometry. However, Grossmann only used chapter 2, which is a "service chapter", introducing, among other things, what we now call Christoffel symbols and the Riemann tensor. Bianchi's presentation follows Christoffel's paper up to notational details, and presents Christoffel's innovations in an accessible manner. ${ }^{21}$

If we put these lines of influence together, the following picture emerges, see Fig. 4. Grossmann largely drew on the tradition of (algebraic) invariant theory, or theory of differential invariants, initiated by Christoffel (1869), and worked out by Bianchi and Lukat (1899), on the one hand, and Ricci and

\footnotetext{
${ }^{20}$ Grossmann in all probability "lifted" the reference from Ricci and Levi-Civita (1901): The reference Grossmann gives is faulty - the page number is wrong - and the identical fault can be found in a relevant passage of Ricci and Levi-Civita (1901). As we know that Grossmann drew heavily on the latter source, it is plausible that he just copied the mistake, and may not actually have consulted Riemann's work at the time of composing the Entwurf. See Räz (2013, Sec. 6.3) for the full argument.

${ }^{21}$ We also observe that Grossmann was Christoffel's successor at the Zurich Polytechnic.
} 


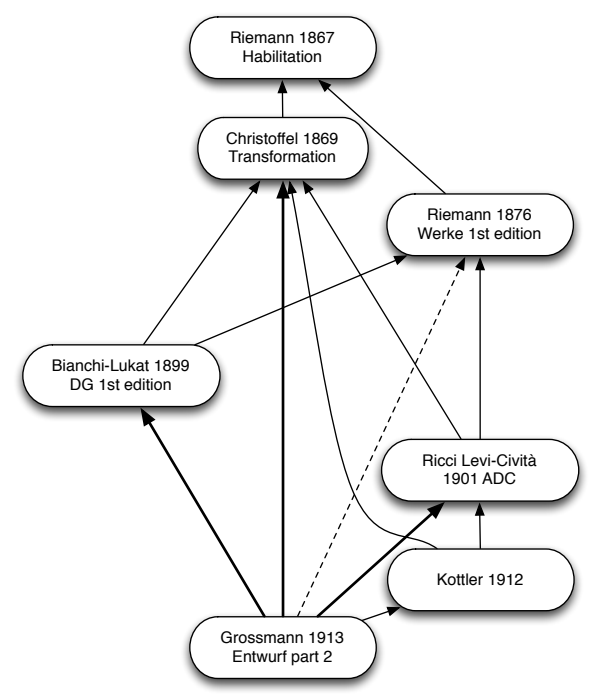

Figure 4: The relative importance of the mathematical sources cited in Grossmann's part of the Entwurf.

Levi-Civita (1901), on the other. The focus was on the abstract calculus, and some particular problems, such as the equivalence of homogeneous quadratic differential forms. On the other hand, the geometrical interpretation of some of the novel concepts, such as the Riemann tensor, were not yet fully worked out, but known only in special cases - the Riemann tensor reduces to Gaussian curvature in two dimensions. The explicit geometrical interpretation of the homogeneous quadratic differential forms as a space-time metric was an exception. The mathematical theory employed at this stage was not differential geometry, as witnessed by the minor direct influence of Riemann (1876). This conclusion is in concordance with Karin Reich's (1992) observation that Riemannian geometry lacked the central concept of parallel transport until after Einstein's final formulation of general relativity when the concept of affine connection was introduced into Riemannian geometry by Hermann Weyl and Tullio Levi-Civita.

Prior Contributions: From Christoffel to the ADC. Let us turn to the prior contributions of pure mathematics, the internal dynamics of mathematics towards application. In the previous section, we have identified the relevant mathematical sources prior to their application to gravitational theory. On 
closer inspection, these sources display a tendency within mathematics that is relevant for the problem of applicability. By comparing the sources in question, we can discern an effort of some mathematicians to make their theories applicable. This can be seen by comparing Christoffel's (1869) paper, on the one hand, and Ricci and Levi-Civita's (1901) on the other.

Christoffel's is a technical paper in algebraic invariant theory. He solves the equivalence problem of homogeneous quadratic differential forms. In a nutshell, he specifies algebraic conditions under which two such differential forms are "essentially the same," i.e., can be transformed into each other by coordinate transformations. This problem is directly relevant for Grossmann's problem: The differential invariants emerging from Christoffel's solution are candidates for the left hand side of the field equations. However, Christoffel's presentation is not easily accessible; his main goal is to solve an abstract, mathematical problem, his solution is cast in a cumbersome technical notation, his aim is not to provide a readily applicable tool.

Ricci and Levi-Civita's paper is entirely different. Their paper advertises the techniques invented by Christoffel, but it explicitly aims at a wide audience of mathematicians and physicists. They take Christoffel's - and other mathematicians' - ideas, develop a calculus, give novel interpretations to the symbolism, and give various possible applications of their calculus. Their paper has the goal of presenting everything in an accessible, yet general manner. They write in the introduction that it is one of their goals to make it easier for the practitioner to get familiar with the methods of a calculus devised to be independent of specific coordinate systems.

This shows that mathematicians do not only strive to solve abstract mathematical problems irrespective of application. There is a distinct tendency within pure mathematics to work towards an application of mathematical theories. If we extend this perspective to Riemann's seminal geometrical work, we get a picture of application cycles that are entirely within mathematics: Riemann's work, which generalized Gauss's work on two-dimensional surfaces to arbitrary dimensions, is a motivation for Christoffel's even more abstract and general work on differential forms, which, in turn, is taken up and transformed by Ricci and Levi-Civita, and applied back to differential geometry by Bianchi and later contributors, closing the application cycle. Mathematics has its own, internal dynamics of application.

Posterior Contributions: Adapting the New Mathematics. Grossmann's contributions to the mathematics of GR, as we find them in the Entwurf, encom- 
pass a spectrum. They range from identifying the appropriate mathematical literature, theories, and concepts, as we have seen, to notational innovations, the reinterpretation of existing concepts, the introduction of new concepts, and the generation of new mathematical results. ${ }^{22}$ These are all contributions that stay within the mathematical domain and are relevant to mathematical theory in itself. Grossmann's contributions are aimed at, but not restricted to, the application to GR.

We can divide Grossmann's part of the Entwurf into two parts. The first three paragraphs are an exposition of what we would now call tensor calculus, an exploration of a mathematical theory, not particularly geared towards any specific application. It is only in paragraph four that specific derivations are carried out, and the theory is put to work for the theory of gravitation. These two parts mirror our distinction between Grossmann's posterior contributions to the development of mathematics, and the deduction steps in the mathematical domain, properly speaking.

Superficially viewed, Grossmann's exposition of tensor calculus in the first part only pulls together the necessary concepts that were provided by the ADC. On closer inspection, Grossman introduced quite some changes in comparison to the original ADC. They provide his posterior contributions. First, Grossmann introduced a change in notation, which may seem odd from our modern perspective. While Ricci and Levi-Civita had denoted their co- and contravariant "systems" by subscript and superscript indices, respectively, Grossmann wrote all indices as subscripts and denoted the character of covariance or contravariance by using Latin or Greek characters for the tensor symbol itself. From a modern perspective, Grossmann's change in notation seems like a step in the wrong direction. However, Ricci and Levi-Civita never considered the possibility of a mixed tensor, i.e. one that carries both covariant and contravariant indices. Grossmann, on the other hand, explicitly introduced tensors of mixed transformation behavior by using Gothic characters instead of Latin or Greek ones, and by separating the co- and contravariant indices with a little vertical line. ${ }^{23}$ Second, Grossmann

\footnotetext{
${ }^{22}$ For a detailed exposition of Grossmann's contributions, see Räz (2013, Ch. 6) and Sauer (2014).

${ }^{23}$ It could be speculated that the generalization of a mathematical concept, viz. the introduction of mixed tensors, reversed a previous notational innovation, the position system for indices, because it was not clear (to Grossmann) whether and how the notation could be consistently generalized along with the concept. Ricci and Levi-Civita, on the
} 
introduced new terminology. He referred to Ricci's and Levi-Civita's "systems" as "tensors", thus reinterpreting one purely mathematical concept in terms of another mathematical concept that had already been endowed with a physical interpretation. Third, Grossmann also contributed new results to pure mathematics; most importantly his proofs that Beltrami parameters (differential operators), and generalizations thereof, can be given a particular form, that is useful to prove, say, the energy-momentum balance equation.

The application of the ADC to a relativistic theory of gravity left its traces in the mathematical domain: the reinterpretation of mathematical notions, such as Ricci and Levi-Civit's (1901) co- and contravariant "systems" as tensors, which was a physical concept previously, the introduction of new concepts that are needed in application, such as mixed tensors and the Ricci tensor; new notation that is motivated or necessitated by the application, such as the distinction between co- and contravariant and mixed tensors using different kinds of letters. Many, but not all of these innovations are now standard in tensor calculus.

\subsubsection{Three Cycles of Applying Mathematics}

The fourth paragraph of Grossmann's part of the Entwurf is divided into three subsections, each presenting the details of a specific mathematical argument, or derivation. In view of the dynamical inferential conception, each of these three parts corresponds to a cycle, the first and third to a closed cycle, the second to an open cycle. We will comment on each of these cycles separately.

It is not surprising that the application of the ADC in GR encompasses more than one cycle, i.e., that the mathematical theory is applied to more than one particular physical problem. Up to this point, it was one problem that pushed Einstein and Grossmann towards the application of the ADC: the generalization of the differential operator acting on the metric. However, once the ADC was established as a more or less suitable tool for this task, the mathematical theory offered itself as a framework in which other aspects of physics could be reformulated as well - mathematics pulled in further applications, so to speak. One such problem is the reformulation of energy conservation, the first cycle to be discussed below.

other hand, did not anticipate the usefulness of mixed tensors; they may have considered the concept overly general. Its introduction was only properly justified by the application in GR. 
The three cycles we find in Grossmann's part of the Entwurf encompass immersion, deduction, and interpretation steps. The deduction step, one of the focal points of the inferential conception, takes place within the mathematical domain. In this step, we draw on a calculus, or on inference rules, in order to extract information about the mathematical structure in question. These inference rules can be implicit or explicit, and are ideally, but not necessarily, deductive. Some deduction steps may not be fully worked out. Material aspects, such as notation, become important, because these can influence how easy or difficult it is to draw inferences - typically these difficulties only become apparent at this stage of concrete operation within the mathematical domain to achieve a certain deduction. In the interpretation step, some of the results of the deduction step are mapped back to the empirical domain, and compared either with empirical results or theoretical background knowledge. This step is not purely mathematical, as empirical and other theoretical considerations come into play - the results of the deduction are brought into contact with the assumed structure.

The First Cycle. In the first argument, Grossmann shows that the energymomentum conservation equation

$$
\sum_{\nu n} \frac{\partial}{\partial x_{n}}\left(\sqrt{-g} g_{m \nu} \Theta_{\nu n}\right)-\frac{1}{2} \sum_{\mu \nu} \sqrt{-g} \frac{\partial g_{\mu \nu}}{\partial x_{m}} \Theta_{\mu \nu}=0
$$

transforms generally covariant under arbitrary coordinate transformations. Here $\Theta_{\mu \nu}$ denotes the contravariant energy-momentum tensor, and $g$ the determinant of the metric tensor. Grossmann's result is appreciated by noting that if we denote the covariant energy-momentum tensor by $T_{\mu \nu}$, covariant differentiation by separating the relevant index with a semicolon, and use the summation convention, eq. (3) translates, in modern notation, to the familiar covariant divergence

$$
\left(\sqrt{-g} T_{m}^{n}\right)_{; n}=0 .
$$

This was an important result, because it establishes that the conservation principle could be written in generally covariant form - the goal of the cycle is the generalization of an existing result.

In the interpretation step, Einstein interpreted eq. (3) as a continuity equation for the energy $(m=4)$ and momentum density $(m=1,2,3)$ by interpreting the first term as a divergence and the second term as expressing the effect of the gravitational force, represented by the derivative 
of the metric, acing on the energy-momentum content. Importantly, while the structure in question, the energy-momentum tensor, had been derived from special-relativistic principles, the general covariance of the generalized energy-momentum balance equation could only be derived using the deductive possibilities provided by the ADC and by Grossmann's preliminary work. Grossmann used the ADC, as well as some of his own results, on the form of differential operators which he had elaborated in paragraph 2 of his part.

This is an example of a closed cycle. Grossmann succeeded in showing that the energy-momentum balance equation carries generally covariant form, as was expected on the grounds of the generalized relativity principle. In establishing this result, he had made substantial use of mathematical properties of the tensor calculus, such as the preservation of the property of general covariance under operations of covariant differentiation. The fact that the cycle was closed, in turn, strengthened the original heuristics of applying advanced and elaborated mathematics as a strategy in the search for a general relativistic theory of gravitation.

The Second Cycle. From the point of view of the dynamical inferential conception, Einstein's and Grossmann's search for a suitable instantiation of the OP slot is an instructive and relevant example of an open application cycle, as it was their original goal to formulate a generally covariant theory of gravitation.

The deduction step of this cycle has two parts. First, Grossmann noted that under the heuristic requirements specified in the immersion step, the Riemann-Christoffel ${ }^{24}$ tensor is the differential operator from which all other possible operators that could enter into the field equation can be generated by algebraic operations. Historically, this is Grossmann's most important contribution to the genesis of GR. This step cannot be overestimated. Reinterpreting the spatio-temporal distance $d s^{2}$ between neighboring events, given in eq. (2), as a differential line-element brings to bear on the problem the rich resources of an elaborated mathematical theory of differential invariants. However, this approach was not yet carried out successfully in the Entwurf.

\footnotetext{
${ }^{24}$ From here on, we will follow Einstein, as e.g. in Einstein and Fokker (1914, pp. 325, 328), Einstein (1914, pp. 1053, 1080), in referring to what is now commonly called the Riemann tensor as the Riemann-Christoffel tensor. By adopting this terminology we do not wish to make a statement on priority but simply to indicate our claim that for Einstein and Grossmann, the source of the expression is Christoffel's work.
} 
To a certain degree, the same may be said of the concepts of space-time metric, and of manifold. It is here that these concepts were put to work for the first time in the context of gravitational theory, but the ramifications and geometrical interpretations of these concepts had not yet been explored.

Second, Grossmann invoked inferential rules provided by the ADC, carrying out concrete formal operations on the level of the symbolic representation, in order to create an object whose representations conformed with the constraints of the field equation frame and its given instantiations. More specifically, the instantiation of the SOURCE slot of the frame (1) of the gravitational field equation by the energy-momentum tensor, a second-rank tensor, created a constraint on the possible instantiations of the OP slot, which also needed to be a second-rank tensor. Grossmann therefore applied the covariant operation of a contraction to the fourth-rank RiemannChristoffel tensor, which produced a second-rank tensor. He thereby created a new mathematical object, the Ricci tensor. The Ricci tensor did not figure in the original ADC as presented in Ricci and Levi-Civita (1901). It appears that Ricci (1904) had already considered what is now called the Ricci tensor, but we have no evidence that Grossmann knew of Ricci's paper; we assume that he found this result on his own. We know from the analysis of the Zurich notebook that Grossmann and Einstein considered further methods of constructing second-rank tensors from the Riemann-Christoffel tensor, ${ }^{25}$ all of which compromised on the original strategy of maintaining general covariance by employing only covariance-preserving operations.

Other commentators on this episode have focused on the next step, the interpretation step in order to explain the failure of this application cycle. The dynamical inferential conception suggests to closely examine the deduction step as well. Grossmann, it must be said, did not exploit all deductive possibilities provided by the ADC. There are other two-index tensors that can be generated from the Riemann-Christoffel tensor in a way that fully preserves general covariance. Consulting the mathematical sources, we find that nothing in the exposition of the ADC in Ricci and Levi-Civita (1901), or in Christoffel's (1869) paper, precluded the addition of a trace term like in the Einstein tensor, or the addition of a scalar term like the later cosmolog-

\footnotetext{
${ }^{25}$ To wit, the construction of the so-called November tensor, which is the part of the Ricci tensor that transforms covariantly under unimodular coordinate transformations, and the further postulation of the so-called Hertz- and $\vartheta$-restrictions; see the discussion in Janssen et al. (2007).
} 
ical term. Grossmann may even have considered non-linear contractions of products of the Riemann-Christoffel tensor without violating the covariancepreserving rules of the ADC. On the other hand, it was not easy to get an overview of the deductive possibilities from the presentation in Ricci and Levi-Civit's (1901) paper, let alone from Christoffel's paper. ${ }^{26}$ Crudely put, this is a failure both of the mathematical literature, which did not make the deductive possibilities sufficiently explicit, and of Grossmann, as he did not fix the problem by trying to get an exhaustive overview of the algebraic possibilities.

We have to turn to the interpretation step if we want to understand why Einstein and Grossmann failed to see that the resources provided by the Riemann-Christoffel tensor actually did provide a suitable mathematization of a relativistic theory of gravitation. In evaluating the Ricci tensor as a possible candidate of an instantiation of the OP slot, we have evidence in the Zurich Notebook (p. 14L) how the interpretation step failed, see Janssen et al. (2007, pp. 610ff). The contraction of the Riemann-Christoffel tensor yielded four terms with second derivatives of the metric, only one of which reduced to the Laplacian on going to the weak-field limit. The other three terms could not be interpreted in terms of the assumed structure of NGT on going to the limit where the assumed structure should be valid. The candidate differential operator, the Ricci tensor, did not yield the Newtonian limit as Einstein expected, and thus violated the main heuristic requirement, the correspondence principle. Thus, the cycle remained open from Einstein's and Grossmann's point of view.

The failure of this application cycle is a famous episode in the history of science. The fact that in their Entwurf, Einstein and Grossmann actually considered the Ricci tensor as a possible tensor to enter into the gravitational field equation, but dismissed this possibility as physically not viable,

\footnotetext{
${ }^{26}$ One of the problems of the ADC paper is that Ricci and Levi-Civita do not state definitions of some relevant concepts. On p. 162, they give the recipe for the construction of "all absolute differential invariants of order $\mu$ ". This recipe relies on the the notions of "absolute differential invariants" and "algebraic invariants". While one can guess what these notions encompass, no explicit definitions are stated in the ADC paper; rather, the reader is referred to other publications. It is not entirely clear how to construct these invariants. Thus, the ADC paper is not self-contained at critical points, despite Ricci and Levi-Civita's stated goal of making the tools of invariant theory available to practitioners; see the mission statement on p. 128.
} 
has puzzled commentators for decades; see, e.g. Pais (1982, sec. 12d). The puzzle got even bigger when John Norton (1984) pointed out that the Zurich Notebook contained a discussion also of the reduced tensor that was taken up later in the first of Einstein's November papers. Closer inspection of the Zurich Notebook then revealed that Einstein and Grossmann even had considered the (linearized) full Einstein equations in their search for a gravitational field equation; see Renn and Sauer (1999). A subsequent detailed line-by-line analysis of the Zurich Notebook, Janssen et al. (2007), revealed the intricate dynamics of Einstein's and Grossmann's applying tensor calculus in their search for a relativistic gravitational field equation.

We have evidence from the Zurich Notebook how Einstein and Grossmann reacted to the failure of the application cycle. They reflected on the steps of the cycle and concluded that the deduction step that produced a differential operator for the OP slot from the Riemann-Christoffel tensor needed to be modified. They introduced additional constraints in order to extract twoindex objects from the Riemann-Christoffel tensor that could be interpreted in terms of the correspondence principle by taking the Newtonian limit. All such attempts failed, since Einstein and Grossmann interpreted the additional constraints realistically as constraints on the gravitational field and not only on its possible representations. ${ }^{27}$ When a number of such attempts had all failed, Einstein and Grossmann gave up on the application cycle altogether and dismissed the theory of differential invariant as a possible mathematical structure for their physics problem.

The Third Cycle. In the third part of the supplement, Grossmann provides the third specific argument, the explicit steps involved in the derivation of the "Entwurf operator". From the point of view of the dynamical inferential conception, this is a deduction step that does not draw on the novel mathematical knowledge provided by the ADC. Rather, Einstein and Grossmann had given up on the possibility of applying covariance preserving mathematical methods altogether and the derivation only used tools of algebra and standard differential calculus.

The strategy now was to go back to the result of the first successful application cycle, the energy-balance equation (3). But instead of further

\footnotetext{
${ }^{27}$ We follow the "majority opinion" of Janssen et al. (2007) of distinguishing between coordinate conditions and coordinate restrictions and interpreting the Hertz- and $\vartheta$ conditions as restrictions. See Norton (2007) for the minority interpretation.
} 
exploiting the resources of the theory of differential invariants, Einstein and Grossmann took the features of the assumed structure as the guiding principle. They now proceeded by inserting a tentative differential operator $\Gamma_{\mu \nu}$ for $\Theta_{\mu \nu}$, which they assumed to have the expected form suggested by the Newtonian limit, and to fix the second-derivatives terms by formal manipulations that only preserved the validity of eq. (3).

The derivation of the Entwurf operator along this strategy yielded a closed application cycle - even though the Entwurf theory eventually proved untenable - because at the time, the formal algebraic manipulations established the Entwurf operator as a uniquely determined object that satisfied almost all heuristic expectations - Newtonian limit and energy-momentum conservation - except for the open question as to its precise covariance group. Two years later, Einstein discovered that his reasoning did not establish the Entwurf operator as the unique solution to his heuristics, an insight that contributed to the demise of the Entwurf theory.

\subsubsection{The ADC Cycles: Taking Stock}

Looking back at the process of applying mathematics in the ADC episode, we can discern the following steps and cycles. The specific problem of finding a differential operator acting on the metric (immersion step) which would take the Poisson equation (assumed structure) to a new, more general field equation (following the heuristics of the principle of generalized relativity) led Einstein and Grossmann to explore the ADC, and its predecessors, mainly as a theory of differential invariants (mathematical domain). Examining the set of generally covariant differential operators, they were able to produce a candidate differential operator, the Ricci tensor, of the right form (deduction step). However, they were unable to produce the right Newtonian limit (failure of the interpretation step, conflict with correspondence principle), and thus failed to find a generally covariant field equation (second cycle subjectively open). Reflecting on the open cycle, they concluded that a generally covariant approach to the field equations may be unfeasible (subjective immersion step mistake), while neglecting some deductive possibilities (objective deduction step mistake), and, most importantly, wrong expectations about some intermediate steps in going to the Newtonian limit (objective interpretation step mistake). This concluded the second cycle. What made the situation more complicated was that, once they had identified the ADC as a suitable framework, the application to other problems, e.g. to the energy balance equation, was successful (first cycle closed). This may have suggested 
that a tensorial formulation of a theory of gravitation would be worthwhile, thus creating a tension between the first and the second cycle. Finally, they were able (driven by the conservation principle) to derive a field equation of restricted, unknown covariance, the Entwurf equations (third cycle closed), which, however, did not draw on the ADC (different mathematical domain). As pointed out before, the Entwurf character can be read off Grosmann's part: The paper only provides an outline of a tensorial theory of gravitation, as the crucial second application cycle remained open for the time being.

\subsection{The Aftermath}

After settling on the Entwurf field equation, Einstein and Grossmann penned their joined publication. Einstein held on to the theory for another two-and-half years, before he gave it up in a dramatic return to general covariance in the fall of 1915, in fierce competition with David Hilbert, see Sauer (1999). This part of the drama is a topic in its own right, and we will not go into details here. On our interpretation, the outcome of the application cycles leading to the Entwurf theory had temporarily stabilized the assumed structure, but it had done so by introducing into the mathematical representation elements that, objectively, were in conflict with and transcended empirical phenomena such as the Mercury perihelion. Over the course of the next two-and-a-half years, changes in the assumed structure were prepared that, in the fall of 1915, led to a renewed attempt of an application of tensor calculus and the theory of differential invariants on Einstein's part that eventually led to a revision of theory which, in turn, enabled a closure of the global application cycle of using tensor calculus for the formulation of a relativistic theory of gravitation.

\section{Conclusions}

The conceptual tools provided by the dynamical inferential conception allow us to analyze the process of the application of mathematics in a descriptively adequate and philosophically insightful manner.

We have identified application cycles as suitable units that constitute the (historical) process of applying mathematics. Depending on their success, they can be open and closed; usually, the construction of a mathematically formulated empirical theory will be an iteration of many cycles, both open and closed, objectively or in the eyes of the working scientists. Cycles can be further analyzed into the components suggested by the inferential conception 
as proposed by Bueno and Colyvan. These components follow their own logic, and, once a cycle is completed, the components make it possible to guide the further course of action in a process of reflection.

We argued that the distinction between assumed structure, the empirical phenomena which are the starting point of the application process on the one hand, and empirical phenomena on the other, is useful. Not all relevant gravitational phenomena are part of the assumed structure - the anomalous precession of Mercury's perihelion did not play a role in the formulation of GR, but rather served as a test for the completed theory.

The distinction between immersion step and an interpretation step, as suggested by Bueno and Colyvan, is instructive. We interpreted the immersion step as the search for a suitable mathematical theory and the embedding of particular empirical phenomena in that theory. The interpretation step brings aspects of the mathematical representation into contact with empirical phenomena.

Based on the historical study, we argued that the mathematical domain itself has to be treated as a separate arena of application. We found that some mathematicians work on abstract mathematical puzzles with negligible ties to direct application, as witnessed by Christoffel's work, while others, such as Ricci and Levi-Civita, strive to make mathematical theories applicable, facilitating inferences, and even suggesting potential domains of application - we called this the prior contributions. We also found that Grossmann himself put a considerable effort into the application of the ADC to gravitation, contributing to many aspects of the mathematical theory - we called this the posterior contributions. The application of mathematics shapes mathematical theories both from within and through the application process.

We examined the various mathematical sources used in the formulation of the Entwurf theory, and we found that it is vital to be precise about the mathematical theory that is actually put to use. In the present case, the importance of an abstract, invariant-theoretic viewpoint should be stressed as opposed to the view that differential geometry was the central mathematical theory for Einstein and Grossmann.

We also sketched the interactions between different application cycles. The second cycle, prompted by the open OP slot in the field equation frame, led Einstein and Grossmann to the ADC. This, in turn, made it possible to reformulate other aspects of the available physical knowledge - the mathematical domain pulls in further applications, as the first cycle shows. However, this created a tension between the cycles, which could not be resolved 
at the time, and was only seemingly fixed in the third cycle, the derivation of the Entwurf field equations.

There are, of course, many open questions and potential for further improvement. We have only begun to explore the larger dynamics that arise if we consider many iterations of application cycles. We would like to say more on the role of reflection on completed cycles, on the different ways in which cycles can be open, closed, on the ways in which cycles can collide and how conflicts can be resolved, and on the different problems that can arise within a cycle, notably the various mistakes in the application steps. We would also like to elaborate on the application dynamics that is directly related to the mathematical theories, for example, on the transformation of mathematical concepts and the corresponding notation - think of Grossmann's problems with the ADC's notation.

In order to further refine the picture we sketched here, it will be useful to consider further episodes in the genesis of GR, particularly if we want to get a better understanding of the long-term dynamics of cycles. Also, the present picture should be extended to other cases from the history of physics. The genesis of QM is a prime example in this respect. Of course, application of the dynamical inferential conception to examples from other sciences should also be carried out.

\section{Acknowledgements}

We would like to thank Claus Beisbart, Michael Esfeld, Hannes Leitgeb, participants of the philosophy of science research seminar in the fall of 2012 in Lausanne, and audiences at the workshop "Metaphysics of science: objects,

relations and structures" in Lausanne, 2012, and at the "Foundations of Physics" conference in Munich, 2013, for comments on earlier drafts of this paper. TR was partially funded by the Swiss National Science Foundation, grant numbers (100011-124462/1), (100018-140201/1).

\section{References}

Bianchi, L. and Lukat, M. (1899). Vorlesungen über Differentialgeometrie. Teubner, Leipzig, 1st edition.

Bueno, O. and Colyvan, M. (2011). An inferential conception of the application of mathematics. Nous, 45(2):345-74. 
Carey, S. (2009). The Theory of Concepts. Oxford: Oxford University Press.

Christoffel, E. B. (1869). Über die Transformation der homogenen Differentialausdrücke zweiten Grades. Journal für die reine und angewandte Mathematik, 70(1):46-70.

Colyvan, M. (2009). Mathematics and the world. In Irvine, A. D., editor, Handbook of the Philosophy of Science: Philosophy of Mathematics, pages 651-702. Elsevier, North Holland.

Earman, J. and Janssen, M. (1993). Einstein's Explanation of the Motion of Mercury's Perihelion. In Earman, J., Janssen, M., and Norton, J. D., editors, The Attraction of Gravitation: New Studies in the History of General Relativity, pages 129-172. Boston, Basel, Berlin: Birkhäuser.

Einstein, A. (1907). Über das Relativitätsprinzip und die aus demselben gezogenen Folgerungen. Jahrbuch der Radioaktivität und Elektronik, 4(4):411462. (Stachel, 1989, Doc. 47).

Einstein, A. (1914). Die formale Grundlage der allgemeinen Relativitätstheorie. Königlich Preußische Akademie der Wissenschaften. Sitzungsberichte, [n.V.]:1030-1085. (Kox et al., 1996, Doc. 9).

Einstein, A. and Fokker, A. D. (1914). Die Nordströmsche Gravitationstheorie vom Standpunkt des absoluten Differentialkalküls. Annalen der Physik, 44:321-328. (Klein et al., 1995, Doc. 28).

Einstein, A. and Grossmann, M. (1913). Entwurf einer verallgemeinerten Relativitätstheorie und einer Theorie der Gravitation. Zeitschrift für Mathematik und Physik, 62(3):225-261. (Klein et al., 1995, Docs. 13, 26).

French, S. (2000). The reasonable effectiveness of mathematics: Partial structures and the application of group theory to physics. Synthese, 125:103-20.

Galison, P. (1997). Image and Logic. A Material Culture of Microphysics. Chicago: University of Chicago Press.

Grattan-Guinness, I. (2008). Solving Wigner's Mystery: The Reasonable (Though Perhaps Limited) Effectiveness of Mathematics in the Natural Sciences. The Mathematical Intelligencer, 30(3):7-17. 
Graves, J. C. (1971). The Conceptual Foundations of Contemporary Relativity Theory. Cambridge, Massachusetts: MIT Press.

Hilbert, D. (1930). Naturerkennen und Logik. Die Naturwissenschaften, 18(47-49):959-963.

Hughes, R. (1997). Models and representation. Philosophy of Science, 64:325-36.

Janssen, M., Renn, J., Sauer, T., Norton, J. D., and Stachel, J. (2007). A Commentary on the Notes on Gravity in the Zurich Notebook. In Renn, J., editor, The Genesis of General Relativity. Vol. 2. Einstein's Zurich Notebook. Commenary and Essays, pages 489-714. Dordrecht: Springer.

Klein, F. (1921). Gesammelte Mathematische Abhandlungen. Erster Band. Berlin: Springer.

Klein, F. (1927). Vorlesungen über die Entwicklung der Mathematik im 19. Jahrhundert. Vol. 2. Berlin: Springer.

Klein, M. J., Kox, A. J., Renn, J., and Schulmann, R., editors (1995). The Collected Papers of Albert Einstein. Vol. 4. The Swiss Years, 1912-1914. Princeton, N.J.: Princeton University Press.

Kottler, F. (1912). Über die Raumzeitlinien der Minkowski'schen Welt. Kaiserliche Akademie der Wissenschaften (Wien). Mathematischphysikalische Klasse. Sitzungsberichte, 121(8-10):1659-1759.

Kox, A. J., Klein, M. J., and Schulmann, R., editors (1996). The Collected Papers of Albert Einstein. Vol. 6. The Berlin Years: 1914-191\%. Princeton: Pinceton University Press.

Ladyman, J. and Ross, D. (2007). Every Thing Must Go. Oxford University Press, Oxford, New York.

Laue, M. v. (1913). Das Relativitätsprinzip. Braunschweig: Friedr. Vieweg \& Sohn.

Minkowski, H. (1908). Die Grundgleichungen für die elektromagnetischen Vorgänge in bewegten Körpern. Königliche Gesellschaft der Wissenschaften zu Göttingen. Math.-phys. Klasse. Nachrichten, pages 53-111. 
Nersessian, N. (2008). Creating Scientific Concepts. Cambridge, MA: MIT press.

Newcomb, S. (1895). The Elements of the Four Inner Planets and the Fundamental Constants of Astronomy. Washington D.C.: U.S. Govt. Printing Office.

Norton, J. D. (1984). How Einstein Found His Field Equations, 1912-1915. Historical Studies in the Physical Sciences, 14:253-316. reprinted in Einstein and the History of General Relativity, eds. D. Howard, J. Stachel (Boston et al.: Birkhäuser, 1989) pp. 101-159.

Norton, J. D. (2007). What was Einstein's Fateful Prejudice? . In Renn, J., editor, The Genesis of General Relativity. Vol. 2, pages 715-783. Dordrecht: Springer.

Pais, A. (1982). 'Subtle is the Lord ...' The Science and the Life of Albert Einstein. Oxford: Oxford University Press.

Pincock, C. (2004). A revealing flaw in colyvan's indispensability argument. Philosophy of Science, 71:61-79.

Pincock, C. (2005). Overextending partial structures: Idealization and abstraction. Philosophy of Science, 72:1248-59.

Räz, T. (2013). On the Applicability of Mathematics-Philosophical and Historical Perspectives. Ph.D. thesis, Université de Lausanne .

Reich, K. (1992). Levi-Civitasche Parallelverschiebung, affiner Zusammenhang, Uebertragungsprinzip: 1916/17-1922/23. Archive for History of Exact Sciences, 44:77-105.

Reich, K. (1994). Die Entwicklung des Tensorkalküls: Vom absoluten Differentialkalkül zur Relativitätstheorie. Historical Studies. Birkhäuser, Basel, Boston, Berlin.

Renn, J. (2006). Auf den Schultern von Riesen und Zwergen. Albert Einsteins unvollendete Revolution. Weinheim: Wiley-VCH.

Renn, J., editor (2007). The Genesis of General Relativity (4 vols.). Dordrecht: Springer. 
Renn, J. and Sauer, T. (1999). Heuristics and Mathematical Representation in Einstein's Search for a Gravitational Field Equation. In Goenner, H., Renn, J., Ritter, J., and Sauer, T., editors, The Expanding Worlds of General Relativity, pages 87-125. Boston et al.: Birkhäuser.

Renn, J. and Sauer, T. (2007). Pathways out of Classical Physics. Einstein's Double Strategy in his Search for the Gravitational Field Equation. In Renn, J., editor, The Genesis of General Relativity. Vol. 1, pages 113-312. Dordrecht: Springer.

Renn, J. and Schemmel, M., editors (2007a). The Genesis of General Relativity, volume 3. Gravitation in the Twilight of Classical Physics: Between Mechanics, Field Theory, and Astronomy. Springer, Dordrecht.

Renn, J. and Schemmel, M., editors (2007b). The Genesis of General Relativity, volume 4. From an Electromagnetic Theory of Matter to a New Theory of Gravitation. Springer, Dordrecht.

Ricci, G. (1904). Direzione e invarianti principali in una varietà qualunque. Atti del Reale Instituto Veneto, 63(2):1233-39.

Ricci, M. and Levi-Civita, T. (1901). Méthodes de calcul différentiel absolu et leurs applications. Mathematische Annalen, 54:125-201.

Riemann, B. (1876). Commentatio mathematica, qua respondere tentatur quaestioni ab Illma Academia Parisieni propositae. In Gesammelte Mathematische Werke und wissenschaftlicher Nachlass, pages 370-383. Leipzig: Teubner.

Rosevaere, N. (1982). Mercury's perihelion. From Le Verrier to Einstein. Oxford: Clarendon Press.

Sauer, T. (1999). The Relativity of Discovery: Hilbert's First Note on the Foundations of Physics. Archive for History of Exact Sciences, 53:529-575.

Sauer, T. (2014). Marcel Grossmann and his contribution to the general theory of relativity. In Jantzen, R. T., Rosquist, K., and Ruffini, R., editors, Proceedings of the 13th Marcel Grossmann Meeting on Recent Developments in Theoretical and Experimental General Relativity, Gravitation, and Relativistic Field Theory. Singapore: World Scientific. [arXiv:1312.4068]. 
Sauer, T. and Majer, U., editors (2009). David Hilbert's Lectures on the Foundations of Physics, 1915-192\%. Dordrecht etc: Springer.

Sommerfeld, A. (1910a). Zur Relativitätstheorie. I. Vierdimensionale Vektoralgebra. Annalen der Physik, 32(9):749-776 ,.

Sommerfeld, A. (1910b). Zur Relativitätstheorie. II. Vierdimensionale Vektoranalysis. Annalen der Physik, 33(14.):649-689.

Stachel, J., editor (1989). The Collected Papers of Albert Einstein. Vol. 2. The Swiss Years: Writings, 1900-1909. Princeton, N.J.: Princeton University Press.

Stachel, J. (2007). The First Two Acts. In Renn, J., editor, The Genesis of General Relativity. Vol. 1, pages 81-112. Dordrecht: Springer.

Steiner, M. (1998). The Applicability of Mathematics as a Philosophical Problem. Harvard University Press, Cambridge Mass., London.

Steiner, M. (2005). Mathematics - application and applicability. In Shapiro, S., editor, The Oxford Handbook of Philosophy of Mathematics and Logic, chapter 20, pages 625-50. Oxford University Press, Oxford.

Suarez, M. (2003). Scientific Representation: Against Similarity and Isomorphism. International Studies in the Philosophy of Science, 17(3):225-244.

Suarez, M. (2004). An Inferential Conception of Scientific Representation. Philosophy of Science, 71:767-779.

Wigner, E. P. (1960). The Unreasonable Effectiveness of Mathematics in the Natural Sciences. Communications on Pure and Applied Mathematics, 13(1):1-14. Reprinted in Philosophical Relections and syntheses, pp. 534549 . 